\title{
Ein Gassystem für die äußeren Spurkammern von HERA-B
}

\author{
Klaus R. F. Bätjer, Oliver Despang
}

\section{Einleitung}

Das Standardmodell zur Beschreibung der Materie und der in ihr herrschenden Kräfte weist einige Lücken auf. Das Experiment HERA-B soll nach dem theoretischen nun auch den experimentellen Beweis dafür liefern, warum Materie und Antimaterie nach dem Urknall nicht in gleichen Anteilen im Universum vorzufinden ist.

Es wird im folgenden ein Gassystem für eine Detektorkomponente von HERA-B, dem „Äußeren Spurkammersystem" beschrieben. Um die Funktion dieses Gasdetektors aufrecht zu erhalten, muissen die 26 Module an ein Gassystem angeschlossen sein, welches in jedem Modul einen Volumenaustausch des Detektorgases pro Stunde und einen Differenzdruck gegenüber der Atmosphäre von $(0 \pm 0,5)$ mbar gewährleistet. Um zu bestimmen, wie ein solches Gassystem ausgelegt werden muß, und ob es möglich ist, mehrere Module mit einer Druckregulation zu betreiben, wurde ein Testgassystem aufgebaut, welches die Bedingungen von HERA-B simulieren soll.

\section{Das DESY-Experiment HERA-B}

Am Deutschen Elektronen-Synchrotron (DESY) in Hamburg werden ab 1999 die Detektoren für das HERA-BExperiment zusammengebaut. Es werden die Wechselwirkungen zwischen beschleunigten Protonen aus der Ringspeicheranlage HERA (Abb. 1) und einem Aluminiumdraht untersucht. Dabei wird ein extrem selte-

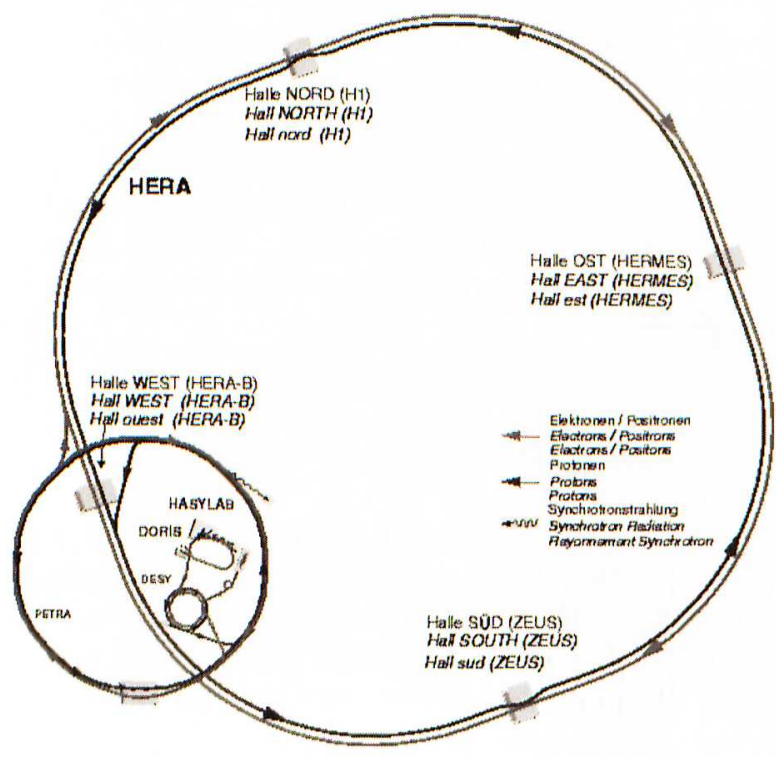

Abb. 1: Teilchenbeschleuniger des DESY ner Zerfall von Teilchen gesucht, der, wenn er nachgewiesen werden kann, in das Standardmodell übernommen wird.

Die Detektoren haben die Aufgabe, ein Teilchen zu identifizieren, dessen Impuls und Energie zu bestimmen, sowie Meßdaten zur Rekonstruktion der Flugbahn zu liefern. Zur Erfassung dieser Meßdaten dient unter anderem das „Äußere Spurkammersystem“.

\section{Die Wabendriftzellen des "Äußeren Spurkammersystems"}

Das „Äußere Spurkammersystem“ ist aus Wabendriftzellen aufgebaut. Jede Driftzelle (Abb. 2) gibt ein Signal, wenn ein geladenes Teilchen sie durchfliegt. Es löst aus

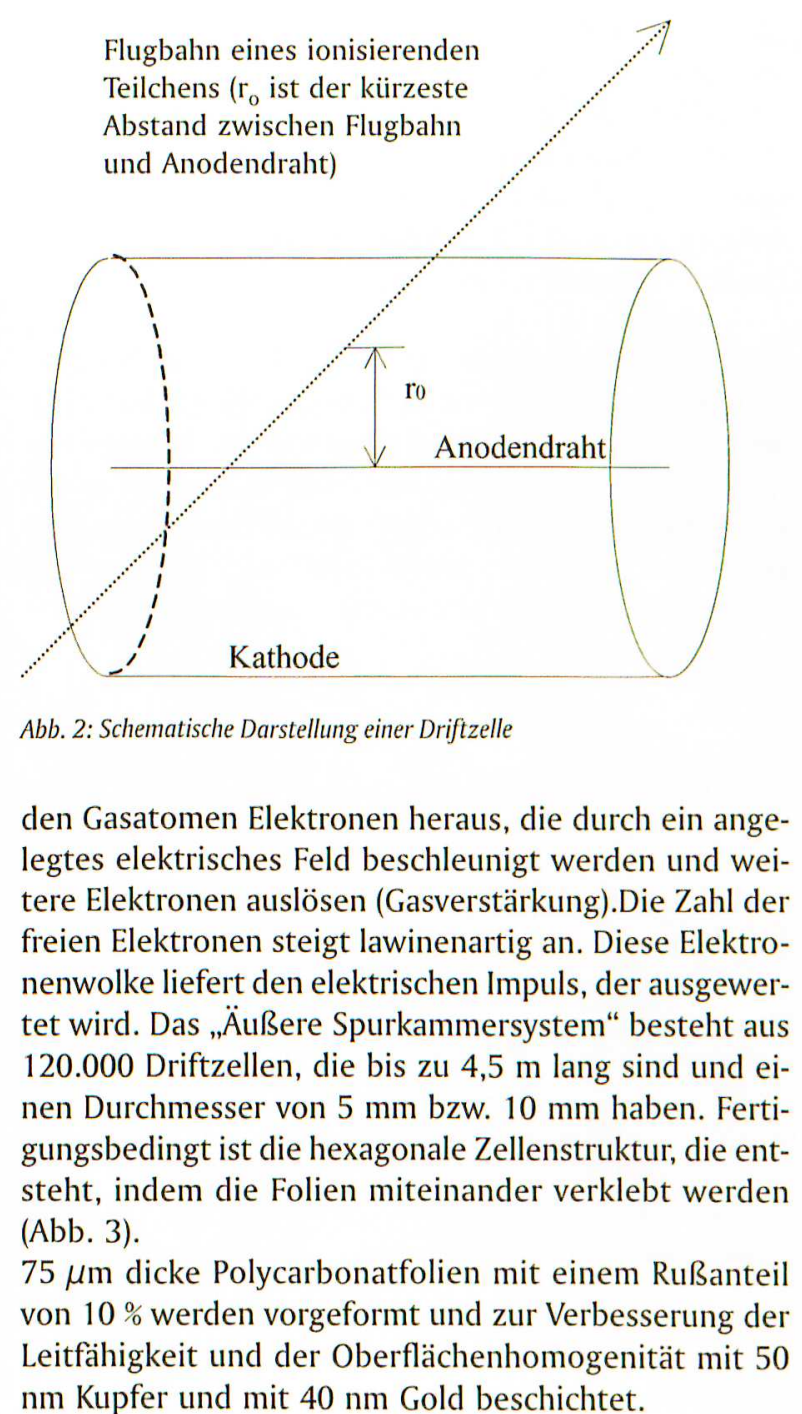

Flugbahn eines ionisierenden Teilchens $\left(r_{0}\right.$ ist der kürzeste Abstand zwischen Flugbahn und Anodendraht)

Abb. 2: Schematische Darstellung einer Driftzelle

den Gasatomen Elektronen heraus, die durch ein angelegtes elektrisches Feld beschleunigt werden und weitere Elektronen auslösen (Gasverstärkung).Die Zahl der freien Elektronen steigt lawinenartig an. Diese Elektronenwolke liefert den elektrischen Impuls, der ausgewertet wird. Das „Äußere Spurkammersystem“ besteht aus 120.000 Driftzellen, die bis zu 4,5 m lang sind und einen Durchmesser von $5 \mathrm{~mm}$ bzw. $10 \mathrm{~mm}$ haben. Fertigungsbedingt ist die hexagonale Zellenstruktur, die entsteht, indem die Folien miteinander verklebt werden (Abb. 3).

$75 \mu \mathrm{m}$ dicke Polycarbonatfolien mit einem Rußanteil Leitfähigkeit und der Oberflächenhomogenität mit 50 nm Kupfer und mit 40 nm Gold beschichtet. 


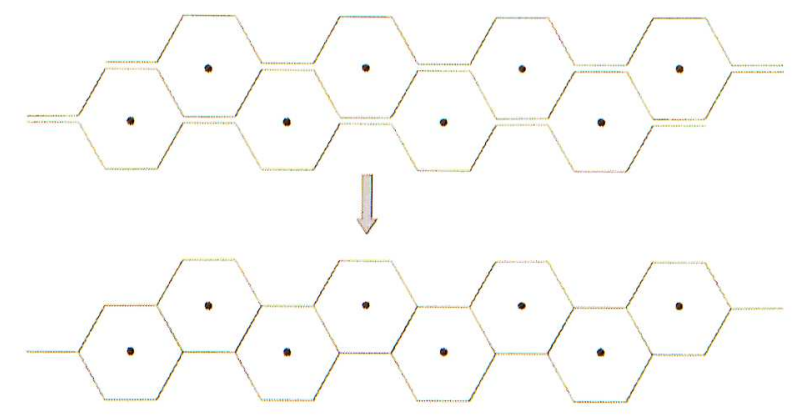

Abb. 3: Schematische Darstellung der Fertigung der Wabendriftzellen

Die Metallschicht der Zellen bildet die Kathode, die Anode ist ein mit Gold beschichteter Wolframdraht von $25 \mu \mathrm{m}$ Durchmesser. Um möglichst große Zählraten zu erreichen, besteht das Zählgas aus einem Gemisch von $65 \%$ Argon (Ar), $30 \%$ Tetrafluormethan $\left(\mathrm{CF}_{4}\right)$ und $5 \%$ Kohlendioxid $\left(\mathrm{CO}_{2}\right)$.

\section{Das Gassystem des "Äußeren Spurkammersystems"}

Bei der Gasverstärkung werden die Bindungen vom $\mathrm{CF}_{4}$ gespalten, und die entstehenden Radikale bilden Polymerketten, die sich im Inneren des Detektors ablagern. Tests haben gezeigt, daß die Signale einer Driftzelle schwächer werden, wenn das Detektorgas nicht ausgetauscht wird. Aus diesem Grund muß der Detektor an ein Gassystem angeschlossen werden, das die Wabendriftzellen ständig mit neuem Gas versorgt. Aus Kostengründen sollte das Gas in einem Kreislauf strömen. Ein Reinigungssystem erhält die Reinheit des Gases, und Verluste durch undichte Stellen werden durch die stete Zugabe von neuem Gas ausgeglichen.

Um die Teilchenflugbahn so wenig wie möglich zu beeinflussen, ist das gesamte Detektorsystem mit möglichst wenig Material gebaut. Dies beeinträchtigt die Stabilität, so daß das Gassystem mit einer sehr guten Druckregulation ausgestattet werden muß. Diese sollte im Inneren des Detektors einen Druck von $(0 \pm 0,5)$ mbar gegenüber dem atmosphärischem Druck konstant halten.
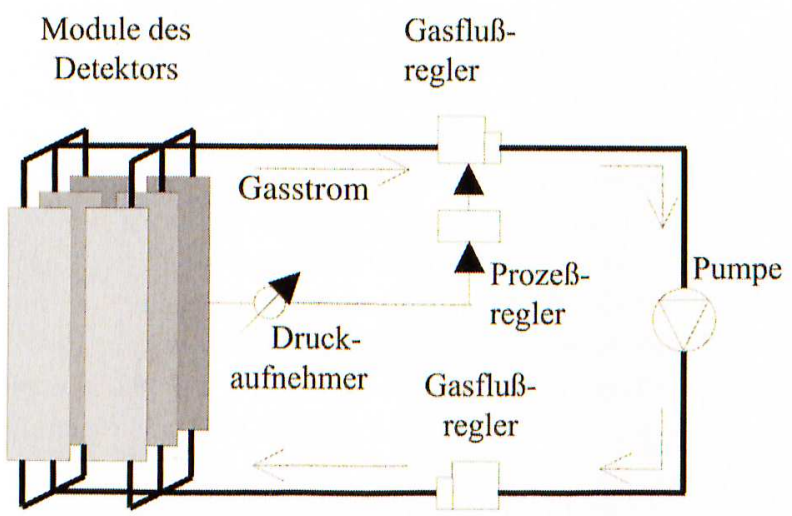

Abb. 4: Schematische Darstellung des Gassystems für das „Äußere Spurkammersystem" (mehrere Module werden mit einer Druckregulation betrieben)

\section{Das Testgassystem}

Das Testgassystem reguliert den Druck in einem Behälter, zwei bzw. vier Behältern und gibt Aufschluß darüiber, mit welcher Genauigkeit mehrere Detektormodule mit einem Druckregelkreis betrieben werden können.

Das Testgassystem hat dieselben Parameter wie das endguiltige Gassystem. Es werden dabei Leitungslängen, Leitungsquerschnitte, Volumenströme und Höhenunterschiede beachtet. Im Test wird ein Gas verwendet, welches mit seinen Eigenschaften dem des beim Experiment eingesetzten Gases so nahe wie möglich kommt, jedoch wesentlich billiger ist: Kohlendioxid $\left(\mathrm{CO}_{2}\right)$ erwies als optimales Gas.

\section{Die Versuche}

Es wurde mit einer einfachen Druckregulation in einem offenen 20-I-Volumen begonnen. Der zweite Versuch fand in einem 40-l-Volumen statt, wobei es sich aber im Gegensatz zum ersten Versuch um einen geschlossenen Gaskreislauf handelte.

\section{Druckregelung in einem 20-1-Volumen}

Die erste Druckregulation findet in einem 20-1-Volumen statt. Um den Aufbau (Abb. 5) einfach zu halten, sind alle Schlauchleitungen so kurz wie möglich.

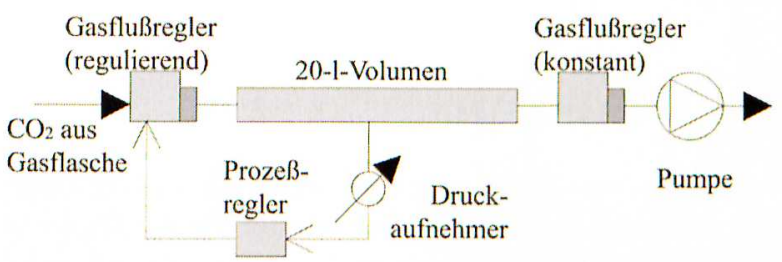

Abb. 5: Aufbau der Druckregulierung in einem 20-l-Volumen

Das Gas einer Druckflasche wird nach dem Durchlauf über eine Pumpe ins Freie geleitet. Ein Gasflußregler am Auslaß hält den Volumenstrom konstant. Ein Druckaufnehmer gibt sein Signal dem Prozeßregler und dieser reagiert, indem er den Volumenstrom mittels des Gasflußreglers am Gaseinlaß reguliert.

Es ergibt sich eine Regelgenauigkeit von $(0 \pm 60) \mu$ bar bei einem Gasdurchfluß von etwa $0,5 \mathrm{l} / \mathrm{min}=30 \mathrm{l} / \mathrm{h}$. Bei den weiteren Tests wird der Volumenstrom am Auslaß geregelt.

\section{Druckregelung in einem 40-I-Volumen}

Der Druck wird in einem 40-I-Volumen, einer ca. $5 \mathrm{~m}$ hohen Aluminiumbox, die innen $30 \mathrm{~cm}$ breit, $3 \mathrm{~cm}$ tief und $4500 \mathrm{~cm}$ hoch ist, geregelt. Die Gasflußregler sind in einer Höhe von etwa $8 \mathrm{~m}$ installiert. Die Abbildung 6 zeigt den Versuchsaufbau. Mit den $50 \mathrm{~m}$ langen Schlauchleitungen werden die Bedingungen von HERA-B beim DESY in Hamburg simuliert. 


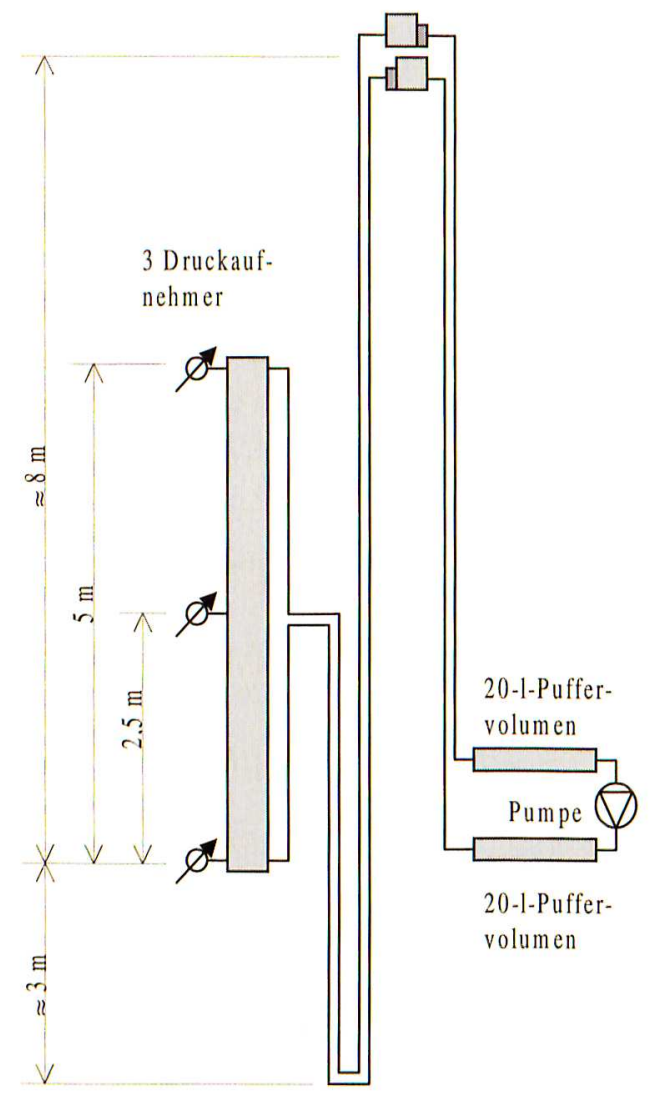

Abb. 6: Versuchsaufbau mit Verlegung der Schläuche bei der Druckregulierung in einem 40-I-Volumen

Die Druckregulierung findet bei einem Volumenstrom von etwa $40 \mathrm{l} / \mathrm{h}$ statt, dabei kann der Druck in der Mitte des Volumens bei $(0 \pm 10) \mu$ bar konstant gehalten werden, siehe Abbildung 7 und 8 .

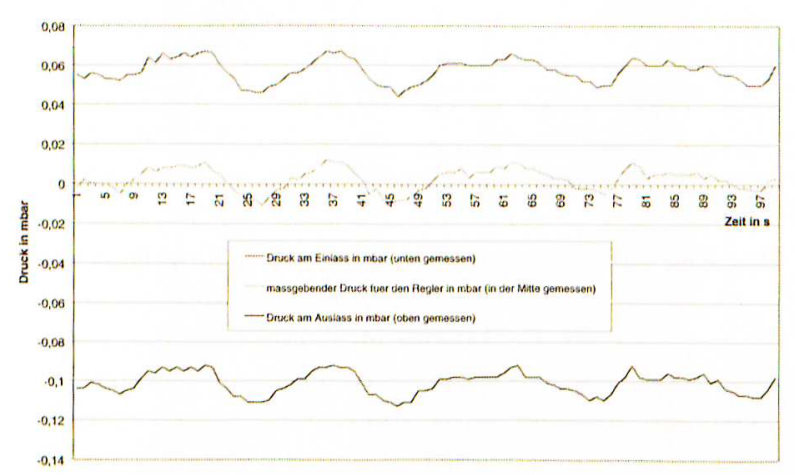

Abb. 7: Druckverlauf bei dessen Regulierung in einem 40-I-Volumen

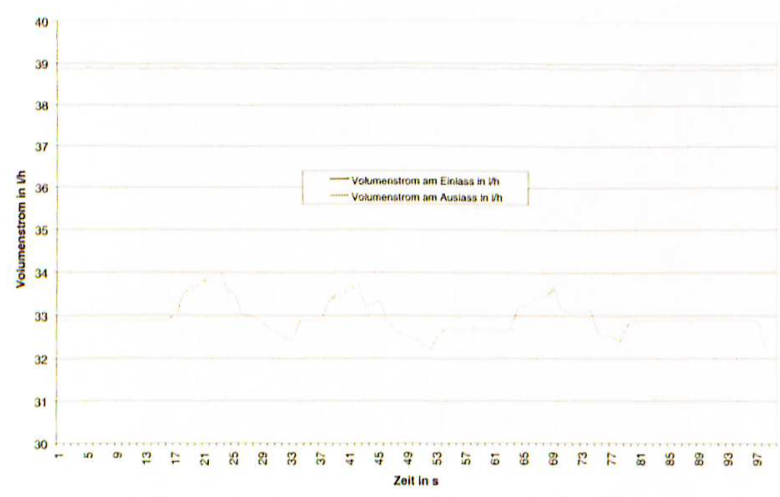

Abb. 8: Verlauf der Volumenströme bei einer Druckregulierung in einem 40-I-Volumen
Bei den Tests mit dem 40-l-Volumen wird der Druck an drei Stellen gemessen. Der Druck in der Mitte des Behälters ist maßgebend für den Prozeßregler, da beim DESY-Experiment der Druck in der Mitte der Superlagenhälften bei $(0 \pm 500) \mu$ bar konstant gehalten werden soll. Bei einem Höhenunterschied von $5 \mathrm{~m}$ ergibt sich ein hydrostatischer Druck von $\mathrm{p}_{\text {hyd }}=(+350) \mu$ bar am Boden des Volumens .

Der tatsächlich gemessene hydrostatische Druck in dem $5 \mathrm{~m}$ hohen Volumen beträgt $170 \mu$ bar und die Druckunterschiede im oberen und im unteren Teil des Behälters sind nicht symmetrisch. Aufgrund undichter Stellen des Systems handelt es sich nicht mehr um reines $\mathrm{CO}_{2}$, sondern um ein Gemisch aus Luft und $\mathrm{CO}_{2}$. Die gesamte Anlage muß mehrmals mit $\mathrm{CO}_{2}$ gespuilt werden.

Diese Vorversuche zeigen, daß in nur einem Volumen der Druck problemlos geregelt werden kann.

\section{Druckregelung in zwei unterschiedlichen Volumen}

Es handelt sich um zwei Volumen von $650 \mathrm{l}$ und $350 \mathrm{l}$. Um in beiden Volumen einen Austausch pro Stunde zu gewährleisten, muß der Gesamtfluß von $1 \mathrm{~m}^{3} / \mathrm{h}$ in die zwei unterschiedlich große Einzelfluisse aufgeteilt werden. Zur Aufteilung in Einzelflüsse stehen verschiedene Varianten zur Diskussion, wobei mechanische Gasflußregler in jeder Zu- und Ableitung (Abb. 9) zur Aufteilung des Gesamtflusses gewählt werden.

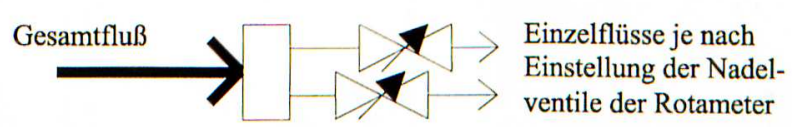

Abb. 9: Asymetrische Aufteilung des Gesamtflusses mittels Rotameter

Obwohl dieser Aufbau höhere Kosten hervorruft, ist der Einsatz mechanischer Gasflußregler, Rotameter, die einzig sinnvolle Entscheidung. Von Vorteil ist die Flexibilität der Rotameter: mit ihnen kann die Aufteilung des Gasflusses bei laufendem Betrieb geändert werden.

\section{Aufbau des Testgassystems}

Das Gassystem besteht aus mehreren Komponenten. Das Gesamtvolumen beider Behälter und der Puffervolumen vor und hinter der Pumpe betragen etwa $1,25 \mathrm{~m}^{3}$. Die Behälter sind $5 \mathrm{~m}$ lange aufrechtstehende Kunststoffrohre mit $30 \mathrm{~cm}$ und $40 \mathrm{~cm}$ Durchmesser, deren beiden Enden geschlossen sind. Der Gasanschluß für die Zuleitung befindet sich unten und der für die Gasableitung oben. Der Druck wird in der Mitte gemessen. Die Puffervolumen sind Kunststoffrohre von je 1201 mit 40 cm Durchmesser und einer Höhe von $1 \mathrm{~m}$.

Die 650-l- und 350-l-Behälter stehen im Erdgeschoß; die Pumpe, der Prozeßregler, die Regelventile und die Ausleseelektronik befinden sich zwei Stockwerke höher. Die Länge der Schlauchleitungen beträgt $50 \mathrm{~m}$ pro Richtung und pro Behälter. 


\section{Das Testgassystem}

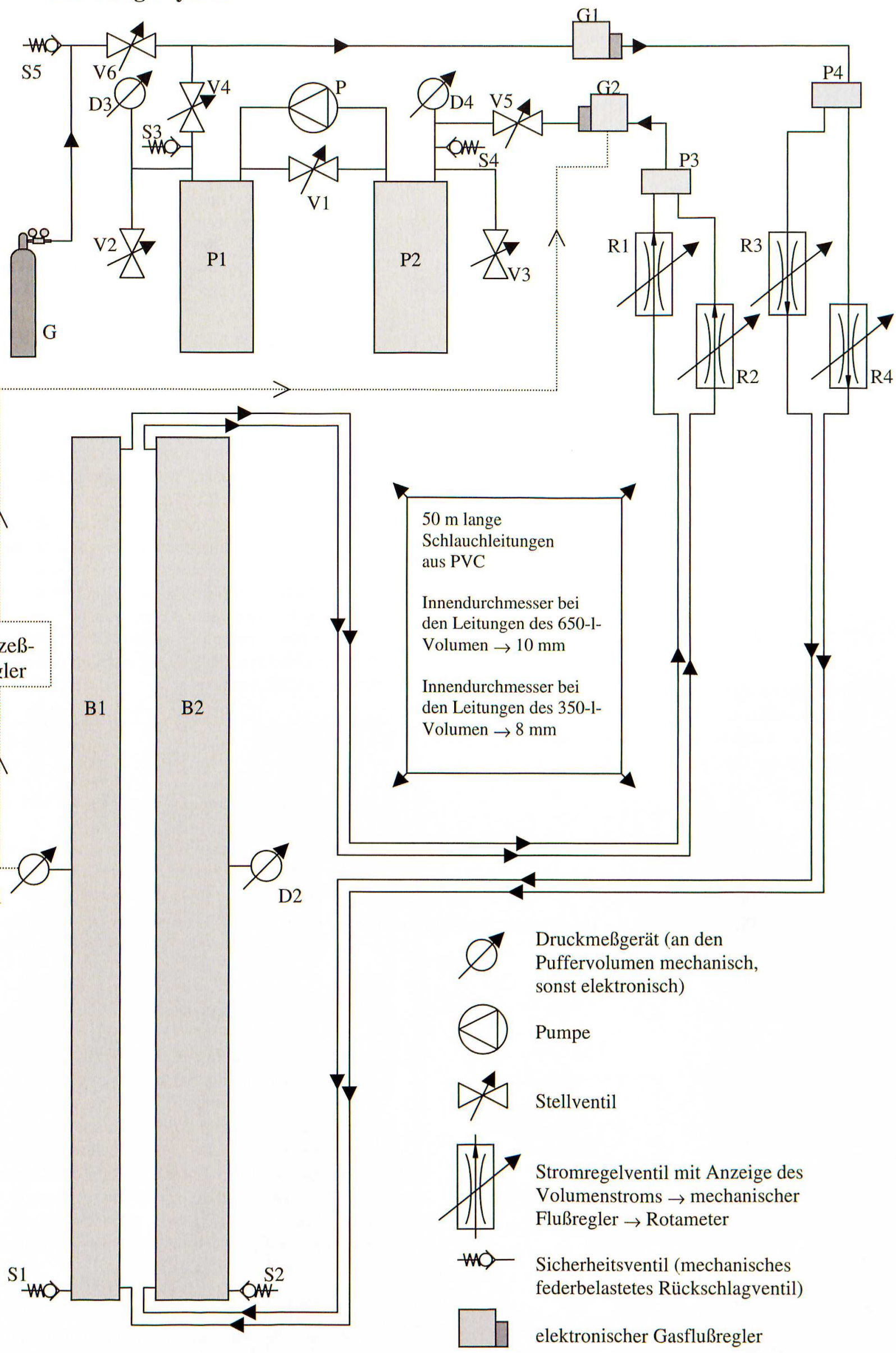

Abb. 10: Schematische Darstellung des Testgassystems 


\section{Sicherheitseinrichtungen}

Sicherheitsvorkehrungen vermeiden einen unkontrollierten Anstieg des Druckes. Der maximale Überdruck der Pumpe beträgt 1 bar, das kann zu einer Belastung von über einer Tonne für die Klebeverbindung zwischen Deckel und Rohr des Volumens ausmachen.

In den beiden Behältern von $650 \mathrm{l}$ und $350 \mathrm{l}$, in denen der Druck durch die Regelung bei $(0 \pm 500) \mu$ bar gehalten werden soll, werden Sicherheitsventile installiert, die sich bei einem Überdruck von mehr als 70 mbar öffnen.

Bei den Puffervolumen liegen die maximalen Drücke (Über- und Unterdruck) der Pumpe an. Die Abbildung 11 zeigt die Anordnung der Puffervolumen.

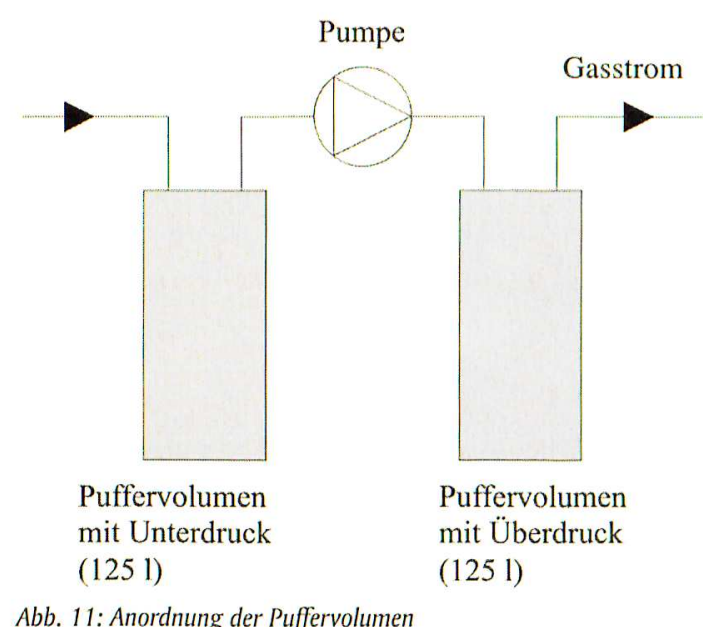

Abb. 11: Anordnung der Puffervolumen

\begin{tabular}{|c|c|}
\hline $\begin{array}{l}\text { Bezeichnung } \\
\text { in Abbildung } 10\end{array}$ & Beschreibung \\
\hline B1 & Behälter mit 3501 Volumen, in dem der Druck geregelt wird \\
\hline B2 & Behälter mit 6501 Volumen, in dem der Druck geregelt wird \\
\hline D1 & $\begin{array}{l}\text { Elektronischer Druckaufnehmer mit einem Meßbereich von }(-10 \text { bis }+10) \text { mbar; } \\
\text { er mißt den Differenzdruck gegenüber der Atmosphäre }\end{array}$ \\
\hline D2 & $\begin{array}{l}\text { Elektronischer Druckaufnehmer mit einem Meßbereich von }(-10 \text { bis }+10) \text { mbar; } \\
\text { er mißt den Differenzdruck gegenüber der Atmosphäre }\end{array}$ \\
\hline D3 & $\begin{array}{l}\text { Mechanisches Barometer mit einem Meßbereich von }(0 \text { bis }+8) \text { bar; er mißt den } \\
\text { Differenzdruck gegenüber der Atmosphäre }\end{array}$ \\
\hline D4 & $\begin{array}{l}\text { Mechanisches Barometer mit einem Meßbereich von }(-1 \text { bis }+7) \text { bar; er mißt den } \\
\text { Differenzdruck gegenüber der Atmosphäre }\end{array}$ \\
\hline G & $\mathrm{CO}_{2}$-Gasflasche $(20 \mathrm{l})$ mit Druckminderer \\
\hline G1 & Elektronischer Gasflußregler; hält den Volumenstrom am Einlaß konstant \\
\hline $\mathrm{G} 2$ & $\begin{array}{l}\text { Elektronischer Gasflußregler; wird vom Prozeßregler (PR) gesteuert und somit } \\
\text { der Druck in B1 und B2 geregelt }\end{array}$ \\
\hline $\mathrm{P}$ & Pumpe \\
\hline $\mathrm{P} 1$ & Puffervolumen (125 l) mit Überdruck mit äußerem Metallrahmen \\
\hline $\mathrm{P} 2$ & Puffervolumen (125 1) mit Unterdruck mit innerer Verformungsbegrenzung \\
\hline P3 & Kleines Puffervolumen $(\approx 21)$ zur Zusammenführung zum Gesamtvolumenstrom \\
\hline P4 & Kleines Puffervolumen $(\approx 2 \mathrm{l})$ zur Aufteilung des Gesamtvolumenstroms \\
\hline PR & $\begin{array}{l}\text { Prozeßregler; liest den Druck in B1 mit D2, vergleicht ihn mit dem Solldruck und } \\
\text { steuert G2 }\end{array}$ \\
\hline R1 & Mechanischer Gasflußregler (Rotameter) mit Anzeige \\
\hline $\mathrm{R} 2$ & Mechanischer Gasflußregler (Rotameter) mit Anzeige \\
\hline R3 & Mechanischer Gasflußregler (Rotameter) mit Anzeige \\
\hline R4 & Mechanischer Gasflußregler (Rotameter) mit Anzeige \\
\hline S1 & Sicherheitsventil mit 70 mbar Öffnungsdruck \\
\hline S2 & Sicherheitsventil mit 70 mbar Öffnungsdruck \\
\hline S3 & Sicherheitsventil mit 1,2 bar Öffnungsdruck \\
\hline S4 & Sicherheitsventil mit 70 mbar Öffnungsdruck \\
\hline S5 & Sicherheitsventil mit 1,2 bar Öffnungsdruck \\
\hline $\mathrm{V} 1$ & Regelventil als Bypass für die Pumpe \\
\hline $\mathrm{V} 2$ & Ventil zum schnellen Belüften von P1; während des Spülens mit $\mathrm{CO}_{2}$ geöffnet \\
\hline V3 & Ventil zum schnellen Belüften von P2 \\
\hline V4 & $\begin{array}{l}\text { Ventil zum Spülen mit } \mathrm{CO}_{2} \text { (V4 geschlossen und V2 offen); Puffervolumen (P1) } \\
\text { kann von der Anlage getrennt werden }\end{array}$ \\
\hline V5 & Ventil zum Trennen des Puffervolumens (P2) von der Anlage \\
\hline V6 & Ventil in der Zuleitung von der Gasflasche \\
\hline
\end{tabular}




\section{Messungen an dem Testgassystem}

Messungen wurden mit verschiedenen Volumenströmen von $250 \mathrm{l} / \mathrm{h}$ und von $1000 \mathrm{l} / \mathrm{h}$ durchgefuihrt.

Die Abbildung 12 zeigt den Verlauf der Drüicke in den beiden Behältern während eines Langzeitversuchs über 5 Stunden.

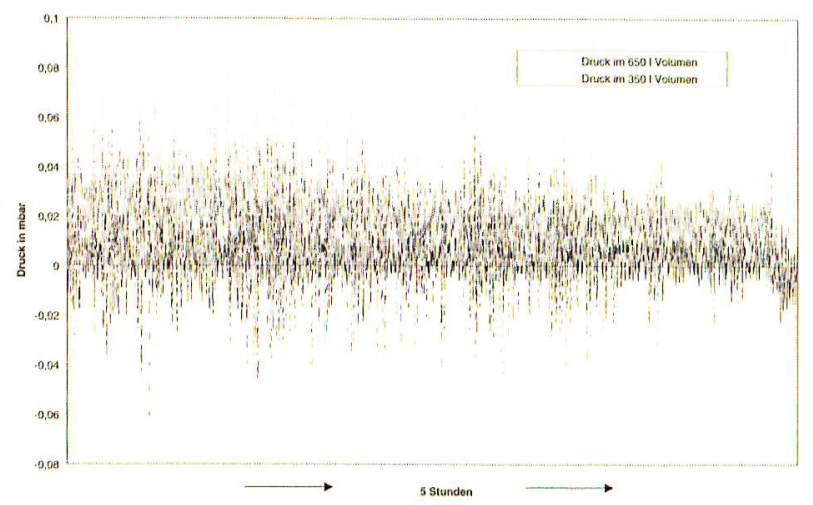

Abb. 12: Druckverläufe bei einem Langzeitversuch über 5 Stunden

Die Abbildung 13 zeigt den Druckverlauf während der Regelung mit einem Volumenstrom von $1000 \mathrm{l} / \mathrm{h}$.

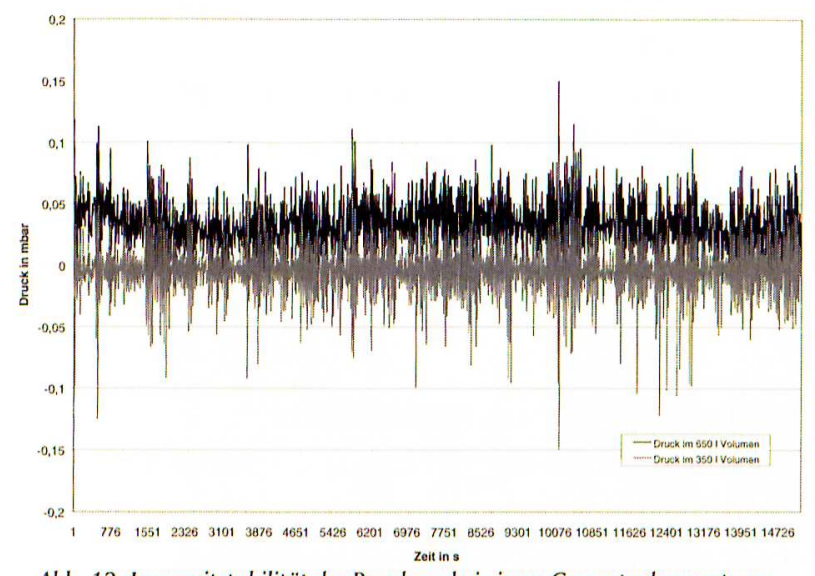

Abb. 13: Langzeitstabilität der Regelung bei einem Gesamtvolumenstrom von $1000 \mathrm{l} / \mathrm{h}$

\section{Schlußfolgerungen aus den Messungen mit dem Testgassystem}

Der Druck kann bei normalem Betrieb in den geforderten Grenzen konstant gehalten werden. Störungen kommen dadurch zustande, daß der Prozeßregler den Druck nur aus einem Volumen liest und der Druck in dem anderen Behälter für ihn nicht zu erkennen ist.

Ein weiteres Problem sind die unterschiedlichen Volumen beider Behälter mit unterschiedlichen Einzelvolumenströmen. Die jeweiligen Gasflüsse werden an den Rotametern am Einlaß eingestellt. Die Rotameter am Auslaß werden danach so justiert, daß der Druck in dem 650-1-Volumen gleich Null ist. Sind die Rotameter einmal justiert, arbeitet die Druckregulation sehr genau. Wird jedoch der Gesamtvolumenstrom verändert, stimmen diese Rotametereinstellungen nicht mehr. Grund dafür sind die unterschiedlichen Strömungswiderstände der Rotameternadelventile. Diese Widerstände verhalten sich bei Änderungen des Gasflusses nichtlinear, woraus die Druckunterschiede nach der Veränderung des Gesamtvolumenstroms folgen. Beim Gassystem des „Äußeren Spurkammersystems“ sollten also die Volumen der Superlagen, die mit einer Druckregulation arbeiten, nahezu identisch sein, damit auch die Einzelvolumenströme und somit die Strömungswiderstände der Nadelventile ähnlich sind.

\section{Das erweiterte Testgassystem}

Nach diesen Testen wird versucht, den Druck in vier Volumen mit nur einem Regelkreis zu steuern. Hierzu werden zwei weitere Kunststoffbehälter mit 1251 und 130 I Inhalt angefertigt und an das Gassystem angeschlossen. Die Abbildung 14 zeigt den Versuchsaubau.

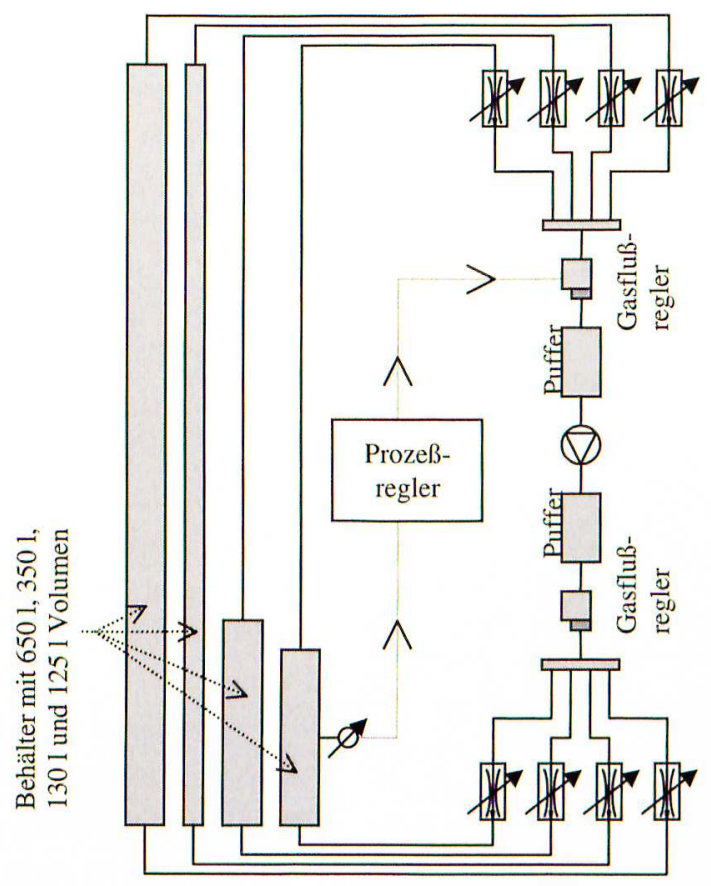

Abb. 14: Schematische Darstellung des Testaufbaus mit 4 Behältern

Der Prozeßregler ist mit dem Druckaufnehmer des 125 l Behälters verbunden, da dieser auf Druckschwankungen am empfindlichsten reagiert.

In der folgenden Abbildung 15 ist die Langzeitstabilität uiber 4 Stunden zu sehen.

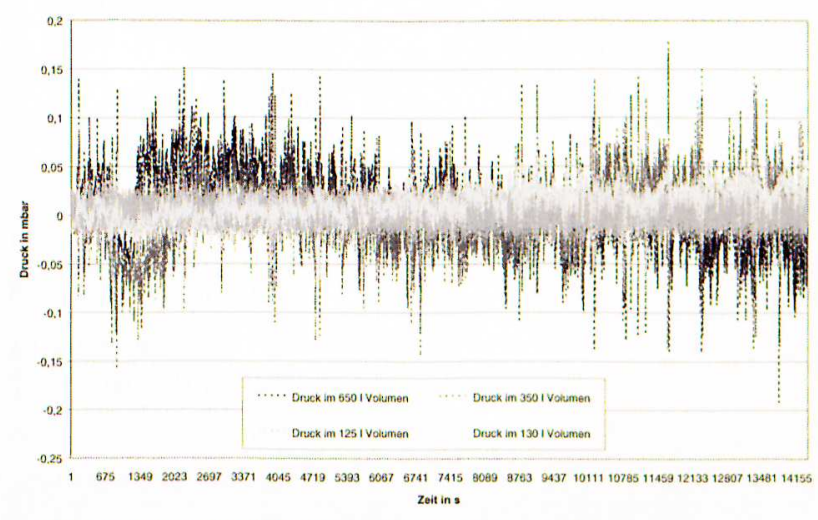

Abb. 15: Langzeitstabilität des erweiterten Testgassystems 
Auch bei dem Testgassystem mit vier Behältern funktioniert die untersuchte Art der Druckregelung. Die erreichte Genauigkeit von $(0 \pm 150) \mu$ bar ist zwar ausreichend, jedoch ist das Einstellen der Rotameter sehr problematisch. Nach der Justierung der Rotameter fuir das $650 \mathrm{l}$ - und $350 \mathrm{I}$ Volumen dauert es bis zu einer Stunde, bis die Drücke in den Volumen konstant bleiben. Bereits sehr geringe Verstellungen der Rotameter führen zu beträchtlichen Veränderungen der Druicke, wobei die vorgegebenen Grenzwerte von $(0 \pm 500) \mu$ bar mehrmals überschritten wurden. An jeder Superlagenhälfte sind damit Sicherheitsventile notwendig, die bei Überschreiten der Grenzwerte von $(0 \pm 500) \mu$ bar öffnen. Damit beim Öffnen der Ventile keine Außenluft eindringen kann, muß der Druckausgleich in einem Puffervolumen erfolgen, in dem sich ebenfalls das Detektorgasgemisch befindet.

\section{Schlußfolgerungen}

Es ist möglich, den Druck in mehreren Volumen gemeinsam mit einer Druckregulation zu steuern, jedoch treten bei beträchtlichen Volumenunterschieden der einzelnen Behälter Probleme auf. Beim Gassystem des „,̈ußeren Spurkammersystems“ sollte daher beachtet werden, daß sich die Volumen der einzelnen Superlagenhälften, die an eine Druckregulation angeschlossen sind, nur geringfuigig unterscheiden.

Bei jeder Änderung des Gesamtvolumenstroms muß der Druck in den Superlagenhälften beobachtet werden und bei zu starker Abweichung vom Solldruck müssen gegebenenfalls die Einstellungen der Rotameter verändert werden.

\section{Autoren}

\section{Klaus R. F. Bätjer}

Technische Fachhochschule Wildau

Fachbereich Ingenieurwesen und

Wirtschaftsingenieurwesen

Bahnhofstraße

15745 Wildau

Tel. (0 33 75) 508-121

\section{Oliver Despang}

Angewandte Plasma-Vakuum-Verfahrenstechnik APVV

Wilhelm-Beckmann-Straße 2

45307 Essen

Tel. (01 73) 2052152 